\title{
(Re)valoración territorial del Gran Pajonal en la construcción de la identidad ashéninka
}

\author{
Territorial (re)assessment of the Gran Pajonal in the Asheninka building \\ identity
}

\author{
Liliana Fernández Fabián
}

Instituto de Investigaciones y Desarrollo Andino (IIDA)

lifernandezf@gmail.com

\section{RESUMEN}

El vínculo entre el fenómeno social y el medio espacial refleja una apropiación simbólica de Oventeni, el centro poblado principal del Gran Pajonal. Esto constituye un cambio del sentido de pertenencia al territorio ashéninka. El objetivo de este artículo es discutir conceptos como lugar, territorio y territorialidad en relación al sentido de pertenencia y su importancia para la construcción identitaria de los jóvenes ashéninkas. Los ashéninkas mantienen un vínculo estrecho entre su quehacer cultural y el dominio de las tierras que conforman su territorio, a pesar que en la actualidad, el desarrollo económico ha transformado la relación entre los lugares que habitan y el proceso de construcción de su identidad o identidades.

Palabras clave: Identidad; territorio; pertenencia; ashéninka; distritalización.

\section{ABSTRACT}

The link between the social phenomenon and the spatial medium reflects a symbolic appropriation of Oventeni, the main populated center of the Gran Pajonal. This constitutes a change in the sense of belonging to the Asheninka territory. The objective of this article is to discuss concepts such as place, territory and territoriality in relation to the sense of belonging and its importance for the building identity of Ashéninka youth, who maintain a close link between their cultural activity and the domain of the lands that make up their territory, although at present, economic development has transformed the relationship between the places they inhabit and the process of building identity and multiples identities.

Keywords: Identity; territory; belonging; asheninka; districtalization. 


\section{Introducción}

Los ashéninkas fueron reconocidos como un pueblo originario por el Ministerio de Cultura en el 2015. Habitan en la Selva Central del Perú, en las regiones de Junín y Ucayali. La mayoría de la población vive en los márgenes del alto Ucayali y en la montaña conocida como Gran Pajonal. Esta última es la zona donde viven alrededor de 7000 personas según la Municipalidad de Oventeni. Se autoidentifican como ashéninkas para diferenciarse de los asháninkas. Esta identidad étnica se fortaleció con el proceso de distritalización del Gran Pajonal (1992-2018) y la oficialización de un alfabeto unificado asháninka (2008). La normalización de tal alfabeto tuvo como consecuencia que los ashéninkas percibieran un abandono del Estado en la educación. En consecuencia, iniciaron reclamos y solicitudes para una normalización particular y se argumentó que ellos eran otro pueblo con otra lengua. Finalmente, el Ministerio de Educación accedió a sus pedidos y brindó soporte técnico para que el pueblo ashéninka consensuara un alfabeto, el cual fue oficializado el 2 de mayo de 2019 con Resolución Ministerial № 199-2019-MINEDU.

Este trabajo plantea el estudio de la (re)valoración del territorio como elemento indispensable para la construcción de la identidad étnica de los ashéninkas, en el marco de las políticas del Estado en la zona limítrofe entre Ucayali y Junín conocida como Gran Pajonal. La posesión del territorio ashéninka ha sido una lucha constante desde que fueron colonizados en un segundo intento, a partir de 1935. Cuarenta años después, los ashéninkas deciden recuperar su territorio y se organizan en comunidades. Con la ayuda logística de organizaciones externas, delimitan su territorio y plantean políticas de desarrollo y apertura al mercado nacional.

Los resultados de esta investigación corresponden a un conjunto de trabajos de campo realizados entre el 2012 y el 2015. Los resultados de este estudio implican un acercamiento temporal y personal a los ashéninkas que han contribuido con esta investigación para comprender mejor el problema del territorio y su importancia en la construcción de su identidad étnica.

\section{Antecedentes}

El libro La Sal de los Cerros de Varese (1973) da cuenta del proceso de colonización en la Selva Central antes y después de la rebelión de Juan Santos Atahualpa. Respecto del Gran Pajonal, se describen las dos etapas dirigidas por los misioneros franciscanos en su labor de evangelizar a «los salvajes» de la Amazonía. Ambas colonizaciones fueron tardías respecto al avance de los franciscanos en otras regiones de la Selva Central, debido a lo inhóspito del territorio y al asentamiento disperso de los ashéninkas.

La primera colonización, en el siglo XVIII, tuvo como objetivo establecer misiones y zonas ganaderas. Hacía 1739, el Gran Pajonal ya era considerado una zona colonizada y próspera. Además, ya se habían instalado 10 puestos misionales franciscanos (Varese, 1973, p. 173). Tras la rebelión de Juan Santos Atahualpa (1742-1756), pasaron más de 100 años para que la colonización y el desarrollo de infraestructura fuesen retomados. A fines del siglo XIX, se reinició la colonización de esta zona, otra vez bajo la dirección de los franciscanos. Los objetivos eran los mismos: el desarrollo de una zona ganadera, la pacificación de los ashéninkas, la colonización e inversión europea y también la construcción de una conexión ferroviaria desde Chanchamayo, a través del Gran Pajonal, hasta Ucayali y los grandes sistemas fluviales (Varese, 1973, pp. 256-263).

En 1935, casi doscientos años después del primer intento colonizador, los franciscanos ingresan con personas procedentes principalmente de Jauja (a quienes los locales llamarán colonos o mestizos, y por antonomasia, a todo aquel que no sea ashéninka). Entonces, fundan Oventeni 
en 1936. De este modo, se inicia un proceso de despojo territorial, servilismo, contiendas y epidemias que desfavorecieron la calidad de vida del ashéninka, diezmando su población.

En la década de los años setenta, surgirán los primeros líderes pajonalinos quienes organizarán a los ashéninkas para formar comunidades nativas y reclamar el derecho a su territorio. Quintori (2003), en Proceso histórico de conformación de la Organización Ashéninka del Gran Pajonal, da cuenta de la conformación de esta organización conocida por sus siglas como OAGP, y sobre la coyuntura sociopolítica de la época.

Hvalkof \& Veber (2005) también abordan la creación de la organización sociopolítica del Gran Pajonal. Señalan que la identidad ashéninka adquirió significado durante el proceso de titulación y reclamo de los títulos de propiedad de sus tierras. La identidad étnica emergida en ese contexto se trasladó también a una autodiferenciación sociopolítica con los asháninkas ribereños.

Fernández (2017), en Construcción identitaria de los jóvenes ashéninkas del Gran Pajonal, resalta la importancia del territorio en la construcción de la identidad étnica y cómo los cambios socioculturales no afectan significativamente el concepto o idea que se tiene del ashéninka «verdadero», ya que la base más fuerte de esta identidad se encuentra en el sentido de pertenencia a cualquier lugar del Gran Pajonal. El sentido de pertenencia, entonces, relaciona sólidamente la territorialidad y la identidad ashéninka. La visión que se tiene del Gran Pajonal y las políticas internas de resguardo de límites dejan ver que esta es la preocupación principal de todo ashéninka y que el grado de seguridad territorial que se tenga influirá en los modos de vida actuales y la construcción de la identidad.

Estos estudios nos permiten comprender cómo el proceso de colonización alteró la vida de los ashéninkas. El liderazgo y organización comunal les devolvió el derecho sobre sus tierras, pero las relaciones de poder entre ashéninkas y mestizos se vio condicionada por el desarrollo económico del Gran Pajonal y el proceso de distritalización. En ese sentido, el territorio es materia de discusión permanente, asunto de vital interés, objeto de posesión y protección.

\section{Proceso de titulación de tierras indígenas}

A partir de la Ley de Comunidades Nativas, promulgada en 1978 (D.L. N. ${ }^{\circ}$ 22175), se reconoce la existencia de los pueblos indígenas amazónicos bajo la personería jurídica de comunidades nativas. La propiedad territorial de estas es reconocida como inalienable, imprescriptible e inembargable (Benavides, 2006; Vilcapoma, 2017). A partir de entonces, las comunidades de cualquier pueblo originario (como se les denomina actualmente a los pueblos indígenas, para evitar connotaciones peyorativas) han tenido que transformarse en personas jurídicas frente al Estado y gestionar el título de propiedad comunal. Este derecho se fue recortando con los años, hasta quitarles, incluso, derechos sobre las áreas forestales y haciendo más burocrática la titulación de comunidades nativas. Así, la Constitución Política del Perú de 1979, en su artículo 57, señalaba que el Estado respeta el uso de las tierras con fines agrícolas por parte de las comunidades campesinas y nativas. La constitución de 1993, sin embargo, retiró el carácter de inajenables e inembargables que tenían las propiedades de las comunidades y solo les dio la calidad de ser imprescriptibles.

La demarcación y legalización de territorios comunales en el Gran Pajonal se inició en la década de los ochenta. En 1988, se firma un convenio entre la Asociación Interétnica de Desarrollo de la Selva Peruana (AIDESEP) y el Ministerio de Agricultura en Pucallpa, para inscribir y titular las tierras de las comunidades nativas. Bajo este acuerdo, se logran inscribir 20 comunidades, las cuales llegan a recibir sus títulos de propiedad. En 1991, se firma otro convenio con el Ministerio 
de Agricultura y se continúa con la demarcación y titulación de comunidades nativas. Esto generó la preocupación de los mestizos de Oventeni, quienes también se involucraron en el proceso para defender las tierras que ocupaban. Hubo muchos conflictos sociales como hostigamientos y amenazas hacia los líderes (Hvalkof \& Veber, 2005; Quintori, 2003).

Se hizo una alianza con los colonos: los ashéninkas se comprometieron a respaldar la titulación de sus parcelas individuales; y aquellos, a no tocar los territorios comunales ashéninkas. Poco después, iniciaría el proceso de distritalización: convertir al Gran Pajonal en un distrito para obtener beneficios del Estado. En 1997, se continúa la demarcación, inscripción y titulación de las tierras comunales faltantes. Hasta el 2002, se titularon un total de 35 comunidades nativas (ver Figura 1). En la actualidad, la OAGP reconoce a 48 comunidades nativas como tales, las cuales fueron formadas en las áreas libres y zonas de amortiguamiento, como pedidos de ampliación y anexos. La motivación para la creación de nuevas comunidades o anexos de las ya existentes, fue el aumento de la población y la creación de escuelas primarias para los niños que tenían que trasladarse distancias largas, entre una y tres horas de camino para recibir una educación formal. Estas nuevas comunidades se encuentran en distintas fases del proceso de titulación. Algunas ni siquiera obtienen la personería jurídica por el gasto logístico y económico que esto implica, pero son reconocidas internamente como comunidades.

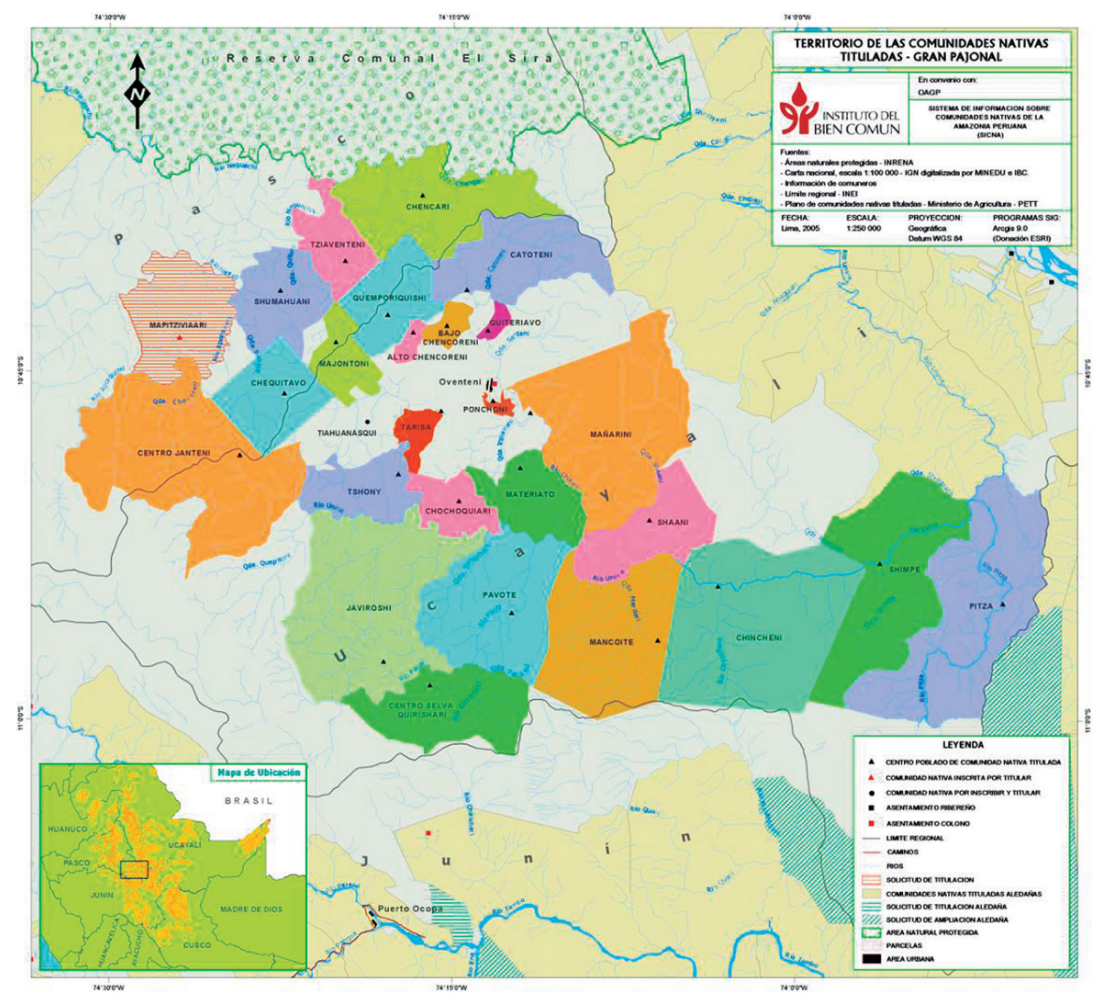

Figura 1. Mapa de las comunidades nativas tituladas del Gran Pajonal Fuente: Benavides (2006)

\section{Proceso de distritalización y relaciones sociopolíticas}

Se trata de un proceso que inician los mismos líderes indígenas que lucharon por la titulación de tierras y reconocimiento de comunidades nativas en el Gran Pajonal. Esta iniciativa nace a finales de la década de los ochenta. Surge a partir de ideas orientadas al progreso de los individuos en el marco contextual de la demarcación territorial al interior del Gran Pajonal y la exigencia de la presencia del Estado en su territorio para elevar el nivel socioeconómico de las comunidades 
nativas. Los líderes ashéninkas y mestizos solicitan a las autoridades locales y nacionales que conviertan su territorio en distrito. Aunque los pedidos eran de ambos grupos socioculturales, la documentación de la OAGP revela que los ashéninkas discutían el proceso internamente en sus congresos y hacían sus propios petitorios (Fernández, 2015).

En 1992, se firman 26 memoriales de las nuevas comunidades nativas de la OAGP, en las cuales solicitan que estas sean reconocidas como anexos para la creación del distrito Gran Pajonal con su capital Oventeni. Estos pedidos son dirigidos al presidente de la Asamblea Regional de Ucayali. En 1996, se crea el Comité de Fundación de la Distritalización del Gran Pajonal con su capital Oventeni. De los siete integrantes, solo 3 eran ashéninkas y el presidente era el médico a cargo de la posta de salud de Oventeni. Dado que no se obtenía respuesta de las autoridades, en 1999, paralelamente a los pedidos de este comité; la OAGP envía un memorial firmado por cientos de comuneros solicitando la creación del distrito al presidente de la república.

A partir del año 2000, se formalizaron y multiplicaron los pedidos. Junto con la Municipalidad del Centro Poblado Menor de Oventeni, la OAGP solicita al presidente del Concejo de Ministros y a la presidenta del Consejo Transitorio de Administración Regional de Ucayali apoyo para la creación política del distrito. De manera independiente, la OAGP enviaría sus propias solicitudes a diferentes entidades del gobierno local, regional y nacional. Finalmente, el 31 de enero de 2003, el Gobierno Regional de Ucayali (GRU en adelante) a través de la Gerencia Regional de Planeamiento, Presupuesto y Acondicionamiento Territorial (GRPPyAT) informa que ha aperturado el Expediente Técnico para la creación del distrito de Gran Pajonal (Informe N. ${ }^{\circ}$ 003-2003-GRU-P-GG-GRPPAT-SGOT-NAS, Oficio N. o 054-2003-GR-Ucayali-P-GGR).

Desde inicios del siglo XXI, se establecerían alianzas con los gobiernos distritales y regionales, gracias a la presencia de regidores ashéninkas en la Municipalidad Provincial de Atalaya, lo cual facilitó el proceso de distritalización. Miguel Camayteri fue regidor en el periodo 2003-2006; Denis Camayteri, en el periodo 2007-2010; Cleofaz Quintori, en el periodo 20112014. No obstante, en octubre de 2009, se informa al entonces presidente de la OAGP, Cleofaz Quintori Soto, que la creación de nuevos distritos y provincias se encuentra suspendida a nivel nacional hasta el 31 de diciembre de 2011, según la Ley N. 28920 (Oficio N.o 360-2009-PCMDNTDT con fecha 07/10/2009).

Finalizado este periodo, en enero del 2012, la Municipalidad de Oventeni solicita al GRU una comisión técnica para la elaboración del expediente de creación del distrito de Gran PajonalOventeni (Oficio N. ${ }^{\circ}$ 005-2012-A-MCPO-GR). Sin embargo, la respuesta no fue positiva, porque Oventeni no reunía los requisitos para convertirse en distrito según la Ley 27795 (Informe N. ${ }^{\circ}$ 045-2012-GRU-P-GGR-GRPPyAT-SGOT). Lo que se exigía era que Oventeni cuente con un plan de desarrollo urbano, la construcción de una plaza pública, la delimitación del mercado de abastos y el cementerio, y contar con una comisaría. Además de iniciar el saneamiento físicolegal de los predios urbanos, era importante que se registre un mínimo de 1800 habitantes en la zona.

Las autoridades locales, tanto colonos como ashéninkas, se preocuparon entonces por modernizar Oventeni, es decir, se debía acondicionar su casco urbano; los habitantes tenían que cambiar de domicilio, ya que muchos habían obtenido su DNI en la región Junín. Era necesario, además, que los ashéninkas se inscriban en el Reniec, dado que la mayoría de la población estaba indocumentada. A mediados del 2012, solo se tramitaron 2660 DNI (Oficio N. ${ }^{\circ}$ 001114-2012/ SGEN/RENIEC). Reniec se trasladó a Oventeni para realizar la campaña, pero los ashéninkas que vivían en las áreas fronterizas del Gran Pajonal no tenían los medios económicos para trasladarse 
masivamente hacia el centro. Las comunidades cerca a Atalaya, se movilizaron hasta la ciudad. Las campañas del Reniec continuaron en los siguientes años, incluso en la actualidad. Las autoridades locales afirman que solo un $80 \%$ de la población pajonalina está inscrita en Reniec.

En 2013, se presenta el Proyecto de Ley N. ${ }^{\circ}$ 2809/2013-CR para declarar de interés nacional y social la creación del Distrito de Gran Pajonal (Proyecto de Ley N. ${ }^{\circ}$ 2809/2013-CR). Las autoridades del Gran Pajonal deciden, entonces, crear un comité que se encargue del proceso de distritalización con el objetivo de mejorar y supervisar las gestiones frente a las autoridades regionales y nacionales. El 30 de diciembre de 2014, se aprueba el Plan de Ordenamiento Urbano del Centro Poblado de Oventeni (Ordenanza Municipal N. ${ }^{\circ}$ 028-2014-A-MPA). Así, se entraría en la última etapa para convertir el Gran Pajonal en un distrito. Quedaría pendiente la definición de delimitación territorial de la provincia de Atalaya con la región Junín, pero se avanza con la apertura del expediente técnico de demarcación y organización territorial de la provincia de Atalaya y de la creación del distrito (Ver Figura 2). Por otra parte, se apertura el empadronamiento de la Lista de adherentes, se verifica el respaldo de la población organizada y se lleva a cabo un diagnóstico socioeconómico (Acta del Comité Pro Distrito Gran Pajonal Oventeni).

Este proceso ha durado 30 años y solo se espera la aprobación del Proyecto de Ley por el Congreso de la República. La documentación revisada, evidencia el trabajo de gestión de los líderes ashéninkas, las alianzas estratégicas para lograr el objetivo de convertir el Gran Pajonal en un distrito, el rol político de los ashéninkas que llegaron a ser regidores, las relaciones de poder entre ashéninkas y colonos o mestizos, y el juego de la memoria y el olvido. A pesar del trabajo en conjunto para lograr este objetivo, ashéninkas y colonos siempre han tenido confrontaciones por el uso de la tierra, el abuso de la mano de obra ashéninka y la discriminación entre ellos. Por eso, asumir la responsabilidad del municipio local ha sido siempre una contienda de poder y orgullo, al menos para los ashéninkas, quienes se reconocen en desventaja frente al dominio del castellano y la administración económica del colono. Cuando no han tenido a cargo el municipio, han constituido una fuerza de confrontación a través de la OAGP, pero para efectos de la distritalización, han sabido unir fuerzas con sus adversarios políticos.

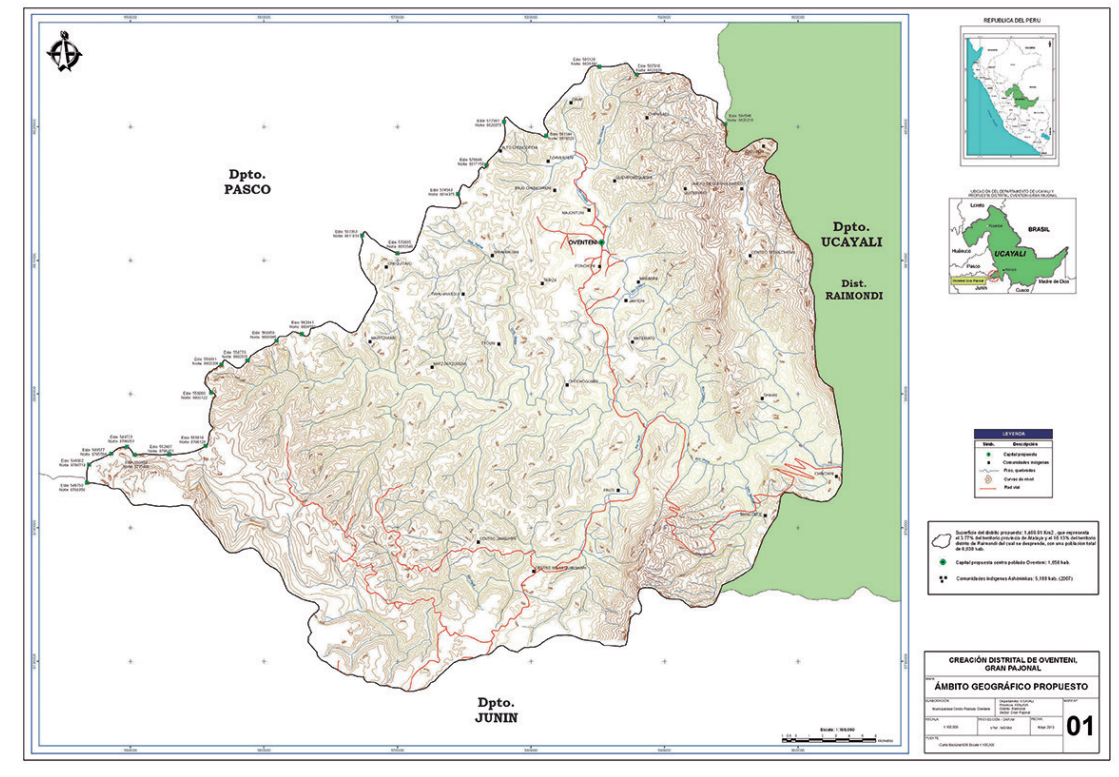

Figura 2. Propuesta territorial para el distrito del Gran Pajonal Fuente: Municipalidad Centro Poblado Oventeni 
Cabe preguntarse ¿qué ocurre con los demás ashéninkas, los hombres y mujeres que viven en las comunidades más alejadas (la distancia entre comunidades puede ser de hasta un par de días caminando)? ¿Qué tan conscientes son los ashéninkas de este proceso y sus implicancias? En primer lugar, ellos luchaban por el reconocimiento de sus comunidades y titulación de las tierras que habían sido usurpadas por los colonos. Sin embargo, el discurso de la distritalización se ha propuesto y manejado desde los líderes de la OAGP. Su aceptación ha estado motivada por los beneficios estatales que tendrían: el acceso a un mercado económico más grande, el desarrollo de infraestructura y sobre todo el acceso a servicios en las comunidades.

En segundo lugar, debemos entender que no todas las generaciones desean el desarrollo económico que supuestamente traerá consigo la creación del distrito. Hay quienes han optado por un estilo de vida tradicional, con una economía de subsistencia, pero la creación del distrito promoverá la agricultura y ganadería como actividades económicas principales y se fomentará la construcción de carreteras al interior del Gran Pajonal. A favor están los jóvenes indiscutiblemente. Las nuevas generaciones aspiran a un desarrollo en infraestructura y servicios que les permita vivir como lo hacen en las ciudades amazónicas que visitan, donde generalmente llegan para estudiar o trabajar por periodos cortos.

Los líderes ashéninkas promovieron la creación de 12 caseríos mestizos en el Gran Pajonal. Esto generó conflictos entre ashéninkas por acusaciones de tráfico de tierras. Muchos ashéninkas consideraron esto como una amenaza de futuras invasiones al interior del Gran Pajonal. Otros pensaron que los líderes estaban vendiendo terrenos comunales. No creían que estos caseríos se habían formado en las tierras libres del Estado.

Esta reorganización del territorio, del keshii, no ha afectado la autoidentificación de los ashéninkas en un sentido negativo. Por el contrario, se ha reforzado para diferenciarse de los colonos o mestizos y de los asháninkas. El territorio es un elemento cultural importante que identifica a los ashéninkas. Este vínculo es la base desde donde se construye la identidad.

Que Oventeni haya crecido visualmente como ciudad capital y ofrezca mejores servicios, lugares de esparcimiento y un mercado económico, ha generado que sea un punto de referencia importante, desplazando a las comunidades nativas en términos de sentido de pertenencia, cuando se trata de autoidentificarse frente al otro, al que no es pajonalino. No obstante, a diferencia de otros centros poblados o caseríos que recién se han formado, la memoria colectiva ashéninka reconoce e identifica Oventeni como un espacio ashéninka, el topónimo mismo favorece esto, ya que Oventeni significa: lugar hondo con quebradas.

\section{Identidad étnica}

Barth (1976) asocia la identidad étnica al concepto de identidad cultural, es decir, a la pertenencia a un grupo social que es distinto a otro culturalmente. Por eso, la identidad étnica se origina y se explica desde las relaciones sociales y políticas que tienen los individuos de distintas culturas. En este contexto, que un grupo étnico conserve o no su identidad es una elección. Así se explica la distinción ashéninka/colono, ashéninka/mestizo. Aunque los intercambios culturales son notorios y hay muchos matrimonios mixtos, la discriminación que se vive en la zona hace que resalten aspectos negativos como diferenciadores y fortalezas positivas como elementos identificadores. Para Cardoso (2007), las relaciones sociales, sobre todo la lucha o reclamo por los derechos, influyen sustancialmente en la formación de identidades, de las cuales la identidad étnica es solo un tipo o clase. Entonces, la etnicidad constituye una herramienta de la construcción identitaria de los ashéninkas frente a los mestizos. 
Es importante conocer este concepto para comprender cómo los ashéninkas han construido su identidad frente a los mestizos de Oventeni y de los caseríos creados en los últimos ocho años en este contexto de luchas y reivindicaciones socio-territoriales. Para Jenkins (2008), la identidad étnica implica diferenciación cultural, así se comparta un mismo territorio. En este tipo de construcción prevalecen las diferencias, no las similitudes. La identidad étnica es una forma de autoidentificación, pero no implica homogeneidad al interior del grupo. Se trata de un proceso dinámico y no estático de las sociedades.

La identidad étnica es un recurso político que surge en situaciones de interacción con sociedades dominantes. En el caso ashéninka, el proceso de titulación de comunidades constituyó un escenario para la revaloración cultural del indígena. Fue una forma de empoderamiento mediante la legalización de la propiedad territorial frente a los colonos. En este caso, todos los ashéninkas tenían el mismo objetivo: salvaguardar su territorio y frenar el avance colono sobre sus tierras. Terrén $(2002$, p. 47) señala que la identidad étnica se relaciona con «la negociación de significados compartidos». La motivación para construir una identidad étnica fue recuperar el derecho al territorio ancestral.

\section{Identidad y territorio}

Las sociedades amazónicas indígenas mantienen una relación trascendental, significativa, entre su quehacer cultural y el espacio donde realizan sus actividades socioculturales. La conceptualización del espacio, de la tierra, de la comunidad, es fundamental para autoidentificarse como ashéninka y ser aceptado como tal por los demás miembros de este pueblo originario. Este contexto de conciencia de lo que implica la territorialidad es la base para la construcción de la identidad ashéninka.

El proceso de distritalización del Gran Pajonal transformó la configuración de este espacio debido a la delimitación geopolítica propuesta. Tras el proceso de reconocimiento y titulación de comunidades nativas, la distritalización segmentó comunidades o dejó algunas fuera del nuevo distrito. Sin embargo, esto no repercutió en el ánimo de los ashéninkas. Al parecer, no se sienten afectados, porque el distrito del Gran Pajonal solo es parte del keshii, pero no es lo mismo. El keshii es el territorio macro que se comprende más allá de fronteras políticas. La creación de anexos, la solicitud de ampliación de tierras y la creación de centros poblados han sido mecanismos en pro de la distritalización y esto ha fortalecido la construcción de la identidad ashéninka, en el sentido que la mayoría de estas personas está pendiente, no solo de sus límites territoriales comunales, sino también de los límites culturales con los vecinos mestizos que han aumentado en la zona y que son vistos como una amenaza, porque el keshii es percibido solo como espacio de pertenencia ashéninka.

El territorio es muy importante para que el ashéninka pueda identificarse ante otros Arawak o quienesquiera. Como señala Fernández (2017, p. 70): «Si un joven se encuentra en la ciudad y dice en castellano: "Soy ashéninka", no ha dado suficiente información para identificarlo. Su enunciado no contiene la información necesaria que lo distinga de otros ashéninkas (de Ucayali, de Yurúa, de Perené, del Gran Pajonal)». Cada ashéninka reconoce su lugar, su geografía, sus recursos y conoce los lugares y recursos de los otros. García \& Surralés (2009), indican que las prácticas culturales son singulares debido a la particularidad de cada territorio donde se han desarrollado los grupos socioculturales. Esta especificidad persona-territorio se hace patente en la modificación del entorno, la utilización de recursos y la toponimia que refleja las motivaciones del ser humano para nombrar su hábitat. 
Esta noción de territorio es intrínseca a la mayoría o todos los pueblos indígenas y así se recoge en el Convenio 169 de la OIT sobre pueblos indígenas y tribales en países independientes, que entró en vigencia en 1991. «Este convenio establece en referencia al territorio que los gobiernos deben respetar la relación cultural y espiritual que tienen sus poblaciones con su tierra, que a su vez incluye el término territorio» (Vilcapoma, 2017, p. 39). En 1994, es ratificado por el Perú mediante Resolución Legislativa N. ${ }^{\circ}$ 26253. Específicamente, los artículos del 13 al 19 se refieren a tierras y territorio. Se considera que los derechos de los pueblos indígenas alcanzan, no solo la superficie terrestre y sus recursos, sino también los recursos del subsuelo.

Los ashéninkas han luchado por el reconocimiento legal de tus tierras y comunidades. La organización política local manejada a través de la OAGP se encargó de liderar y concientizar a los ashéninkas sobre el poder que tienen como personerías jurídicas. Así, el proceso de titulación generó el fortalecimiento de la institucionalidad y de la construcción de la identidad ashéninka. Como sostiene Bello (2004, p. 95), el territorio no solo es la «base de sustentación económica, sino también [...] fundamento de su identidad, sus sistemas de vida y, sobre todo, sus proyectos de autonomía». Esto nos ayuda a entender por qué algunos ashéninkas encabezados por los líderes pajonalinos apostaron por la creación del distrito del Gran Pajonal.

\section{Kipatsi, keshii y Oventeni}

Según García \& Surralés (2009, p. 24), el territorio «es el punto de partida y el resultado actualizado de la historia y la cultura de un pueblo». En ese sentido, debemos entender que la división del mundo ashéninka soslaya los conceptos occidentales relacionados a lugar, tierra y territorio. Para los ashéninkas, el espacio, el lugar, no es solo físico. El kipatsi es el centro del mundo, es la tierra, es un espacio a través del cual se conectan otros mundos posibles y paralelos a aquel que habita el atziri «gente».

El keshii es el etnónimo ashéninka para referirse al Gran Pajonal. Está dentro del kipatsi, concepto amplio y variado que implica tanto espacio como territorio y temporalidad. Esto se debe al vínculo estrecho que han creado los pueblos indígenas con su lugar de origen. Kipatsi hace alusión al elemento material que se puede tocar, pisar, mirar. Pero también va más allá cuando se comprende en la línea temporal como el espacio que es resultado de la presencia milenaria de un pueblo que ha modificado su entorno y ha puesto en ese espacio el valor ontológico a sus manifestaciones culturales. «Para un ashéninka, kipatsi es un todo diacrónico en donde se reencuentra con sus antepasados, donde la existencia y la vida cobran sentido» (Fernández, 2017, p. 71).

A los habitantes del Gran Pajonal se les denomina keshiijatzi. El keshii es una meseta cuya elevación varía entre los 800 y los 1500 m.s.n.m. (Quintori, 2003, pp. 18-19). Se ubica en la parte suroeste de la región Ucayali entre las regiones de Junín y Pasco y los ríos Ucayali, Tambo, Perené y Pichis. Limita con la reserva comunal El Sira por el norte; con los distritos de Rio Tambo y Rio Negro (Satipo, Junín) por el sur; con el distrito de Rio Tambo (Satipo, Junín) y distrito de Raimondi (Atalaya, Ucayali), al cual pertenece, por el este; con el distrito de Puerto Bermúdez (Oxapampa, Cerro de Pasco) por el oeste (Véase la Figura 3).

Aproximadamente en el centro del Gran Pajonal, se ubica el pueblo Oventeni, topónimo ashéninka que significa hondura con agua (ver Figuras 4, 5 y 6). Fue fundado en 1936 y será la capital del distrito Gran Pajonal. Existen dos vías de acceso. Desde Satipo, por la carretera que va desde Puerto Ocopa a Oventeni (7 horas en camioneta). Desde Atalaya, por la carretera que se dirige a Puerto Ocopa, se detiene en Unini, en la zona conocida como Repartición y de ahí dobla 
hacia Oventeni (7 horas en camioneta). Alrededor de Oventeni, se elevan pequeñas montañas que pertenecen a las comunidades nativas Mañarini, Ponchoni, Quiteriavo, Bajo Chencorini.

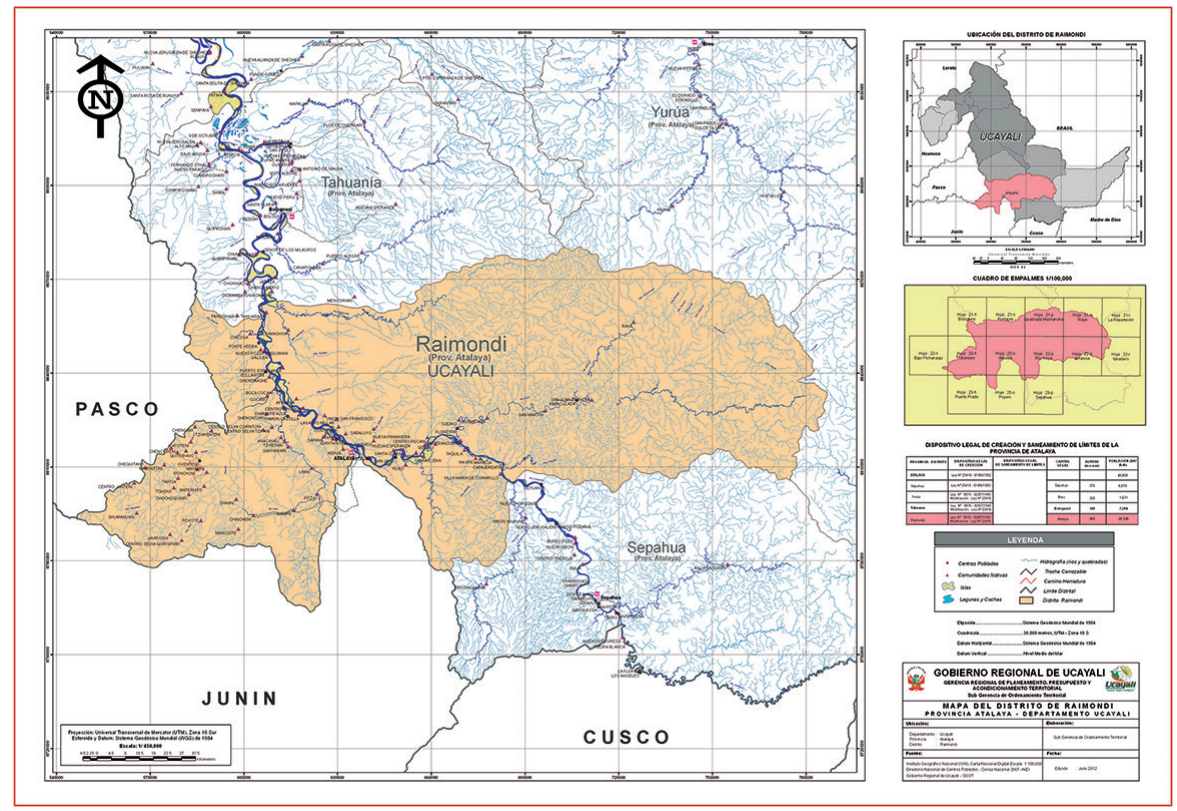

Figura 3. Mapa del distrito de Raimondi Fuente: Gobierno Regional de Ucayali

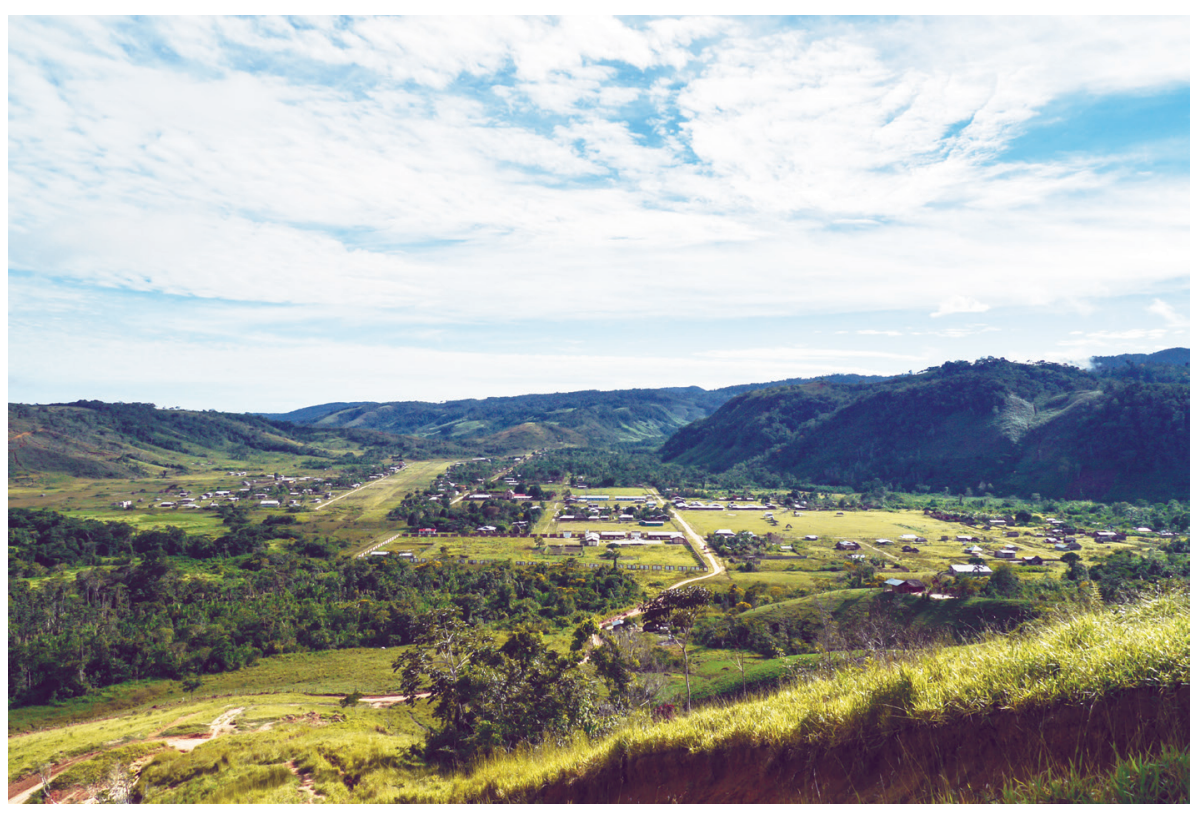

Figura 4. Vista general de Oventeni Archivo Liliana Fernández, 2012. 


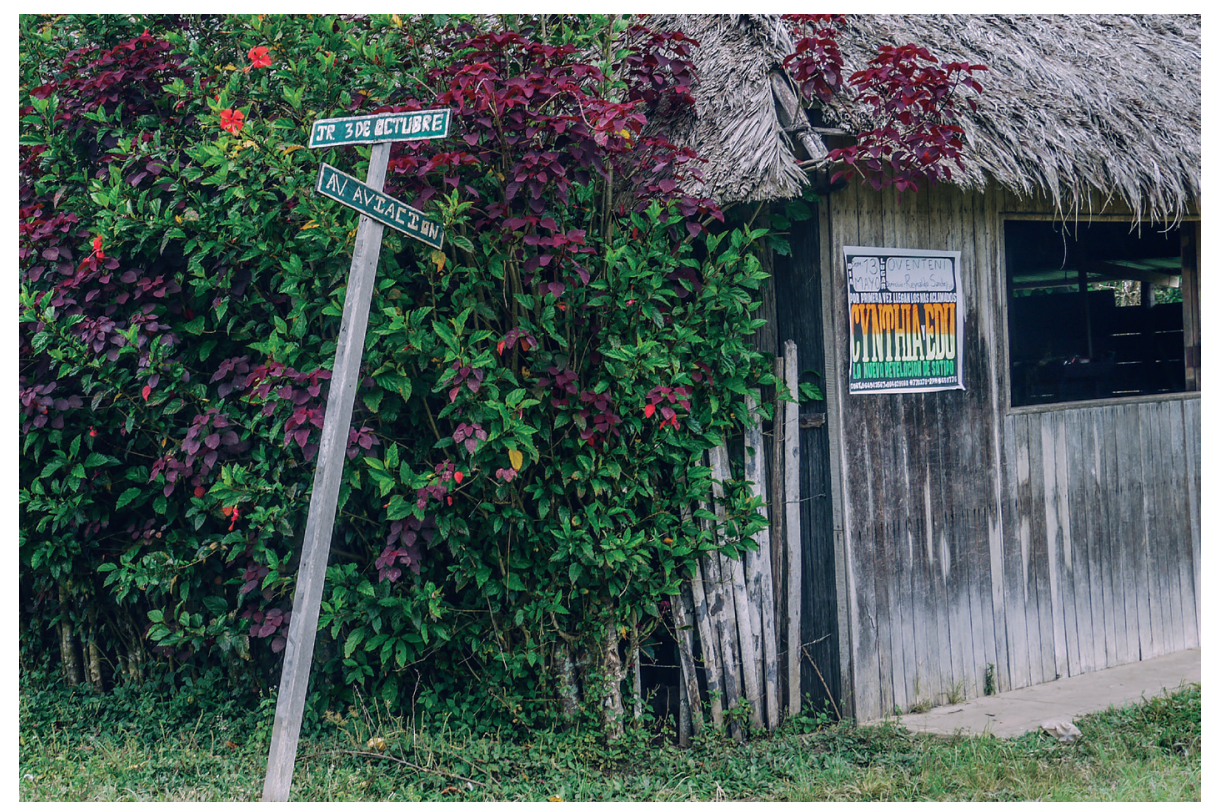

Figura 5. Intersección de la av. Aviación y el jr. 3 de octubre Archivo Liliana Fernández, 2012.

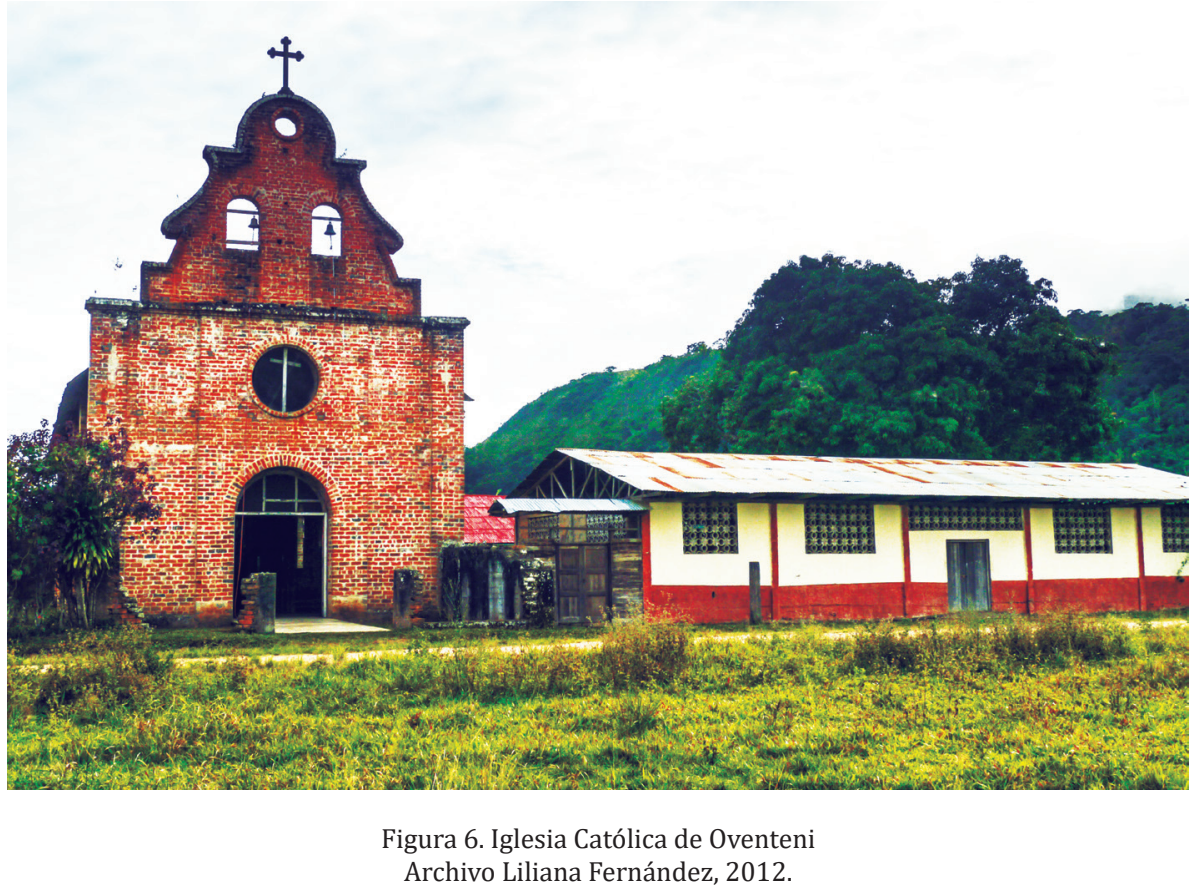

En Oventeni, se encuentran las principales instituciones estatales como la posta de salud, la oficina de la red educativa, una oficina del Ministerio de Agricultura, la oficina de los ronderos, la iglesia, y la Municipalidad. Oventeni cuenta con un mercado que cubre las necesidades básicas de los pajonalinos (véase Figuras 7 y 8). Las tiendas de abastos son pequeños supermercados donde se puede encontrar de todo. Además hay una botica y una panadería. El contraste con el ordenamiento de una comunidad es notorio. Oventeni se ha transformado en una ciudad, sobre todo desde el año 2012 con el fin de lograr la distritalización del Gran Pajonal. 


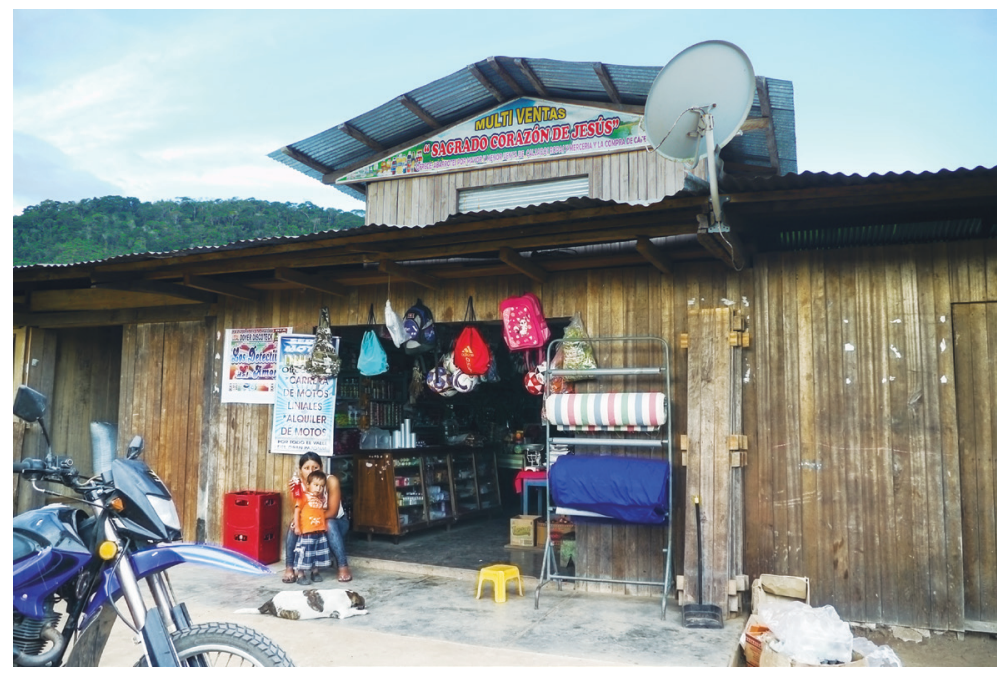

Figura 7. Tienda en el mercado de Oventeni Archivo Liliana Fernández, 2012.

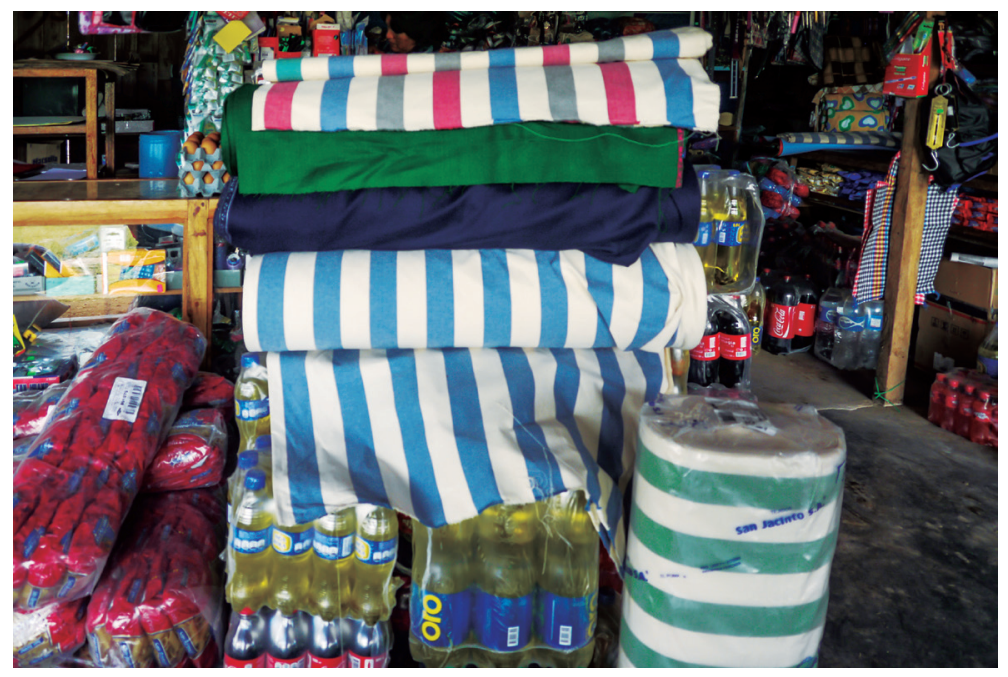

Figura 8. Productos que se pueden encontrar en Oventeni Archivo Liliana Fernández, 2012.

Como centro económico, Oventeni tiene un gran movimiento comercial durante la cosecha de café y principalmente los domingos. Es el lugar donde se encuentra el terminal de camionetas que llevan y traen pasajeros que se dirigen a distintas comunidades nativas. Aquí se encuentra el complejo deportivo donde el fútbol es el pretexto para socializar los domingos. Existe una liga de fútbol y ashéninkas de distintas comunidades llegan para participar con sus equipos.

\section{El sentido de pertenencia}

Es el vínculo que une a una persona con un espacio geográfico determinado. Generalmente, se trata del lugar donde nació o creció, del lugar en el cual forma parte de una colectividad. Los ashéninkas dicen que son del Gran Pajonal cuando hacen referencia a su comunidad nativa o anexo donde viven ellos o su familia. El sentido de pertenencia es una forma de apropiarse de un lugar y un medio para construir la identidad.

El prestigio y fácil referencia de Oventeni en las ciudades de Satipo y Atalaya, por ejemplo, ha motivado esa pertenencia ashéninka a la futura capital del nuevo distrito. No se 
trata de un arraigo vinculado a lo comunal, ya que Oventeni resulta hostil para el ashéninka en términos de relaciones personales con los mestizos. Por eso, este sentido de pertenencia se da hacia el exterior, pero dentro del Gran Pajonal la referencia primera es la comunidad nativa, el nampitsi. Muchos ashéninkas se han mudado a Oventeni, pero mantienen vínculos con su comunidad nativa: ahí tienen su chacra, participan en las faenas y reuniones. Otros, en cambio, ya no tienen comunidad.

En el contexto pajonalino, el sentido de pertenencia es subjetivo y puede cambiar; no es estable, depende de las relaciones sociales de los individuos. La coyuntura del proceso de distritalización ha propiciado que Oventeni se eleve a modelo de desarrollo para las comunidades nativas. Debemos tener en cuenta, sin embargo, que Oventeni es solo una parte del keshii y este sentido de pertenencia se observa en los ashéninkas que viven en este sector; en tanto los ashéninkas del sector carretera mantienen como referencia única al keshii y no se identifican con Oventeni (ver Figura 9). Dado que los problemas limítrofes del Gran Pajonal (invasiones de colonos, de ashéninkas, de terroristas) involucran y pueden perjudicar a todos los ashéninkas; el keshii como territorio es más importante que Oventeni.

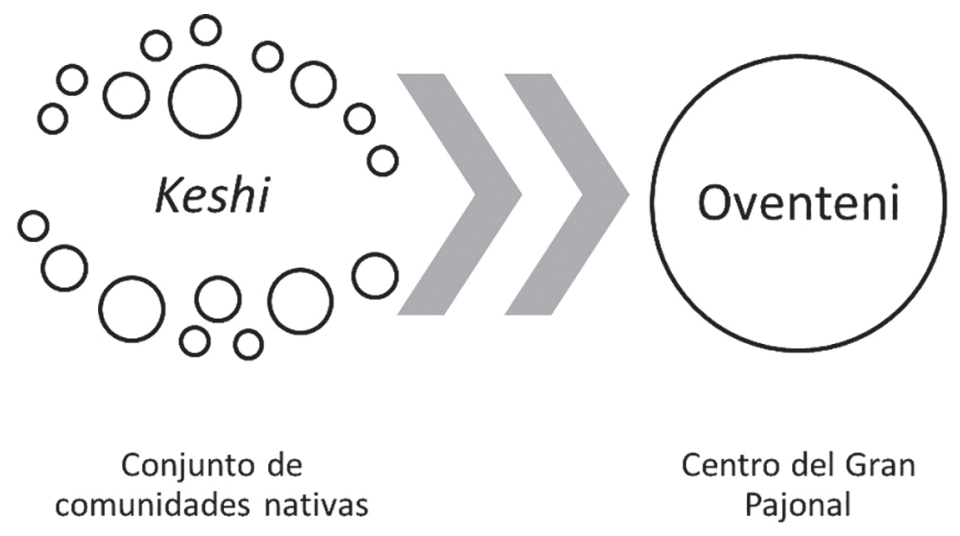

Figura 9. Orientación del sentido de pertenencia Fuente: Fernández (2017, p. 79)

La mayor significación de un lugar u otro dentro del keshii no afecta la valoración del territorio en su conjunto, tampoco los cambios que ha sufrido el paisaje producto de nuevas actividades económicas como el cultivo del café o el avance de la carretera al interior del keshii. No obstante, la preferencia por determinados lugares sí afectará la construcción de la identidad de los sujetos. El arraigo o desarraigo generará identidades múltiples de acuerdo a las relaciones de poder que establezcan las personas.

\section{Desarrollo económico y territorio}

El territorio del Gran Pajonal se ha visto modificado, aunque levemente, por el impacto de los proyectos de inversión y fomento de la agricultura. El avance de la carretera hacia el interior de las comunidades para extraer madera y los puentes sobre los ríos y quebradas han ocasionado la alteración del antamimashi «bosque» (ver Figuras 10, 11 y 12). Asimismo, la comunidad ha cambiado el patrón de asentamiento, la arquitectura tradicional ha incorporado materiales occidentales y las nuevas necesidades obligan a los ashéninkas a generar una fuente de ingreso económico, principalmente a través de la siembra, cosecha y venta del grano de café. 


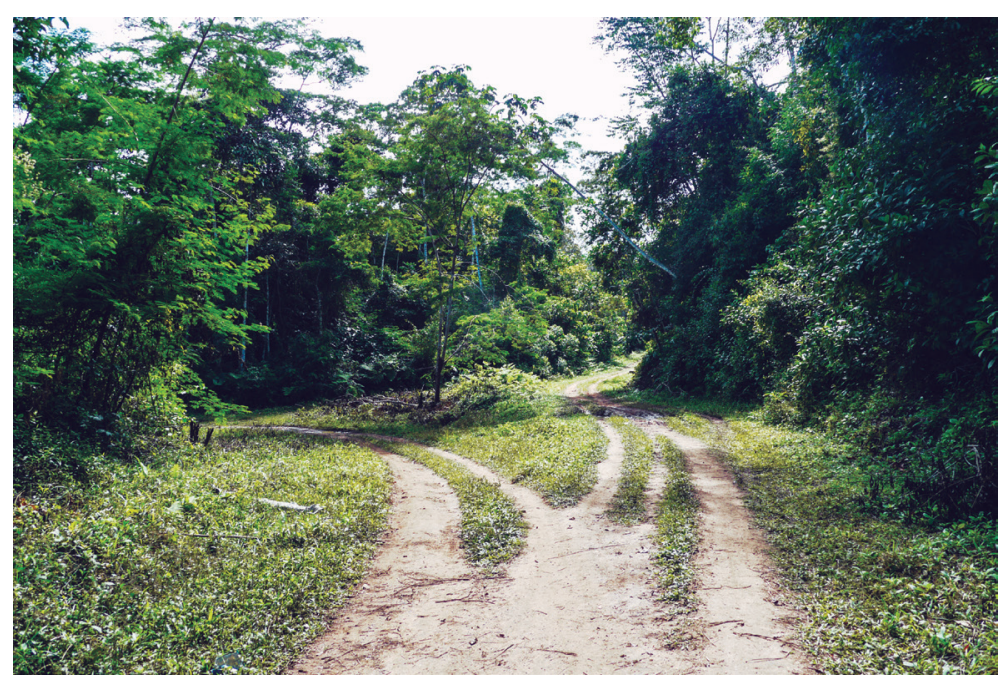

Figura 10. Bifurcación de la trocha hacia Catoteni y Kirahuanero Archivo Liliana Fernández, 2012.

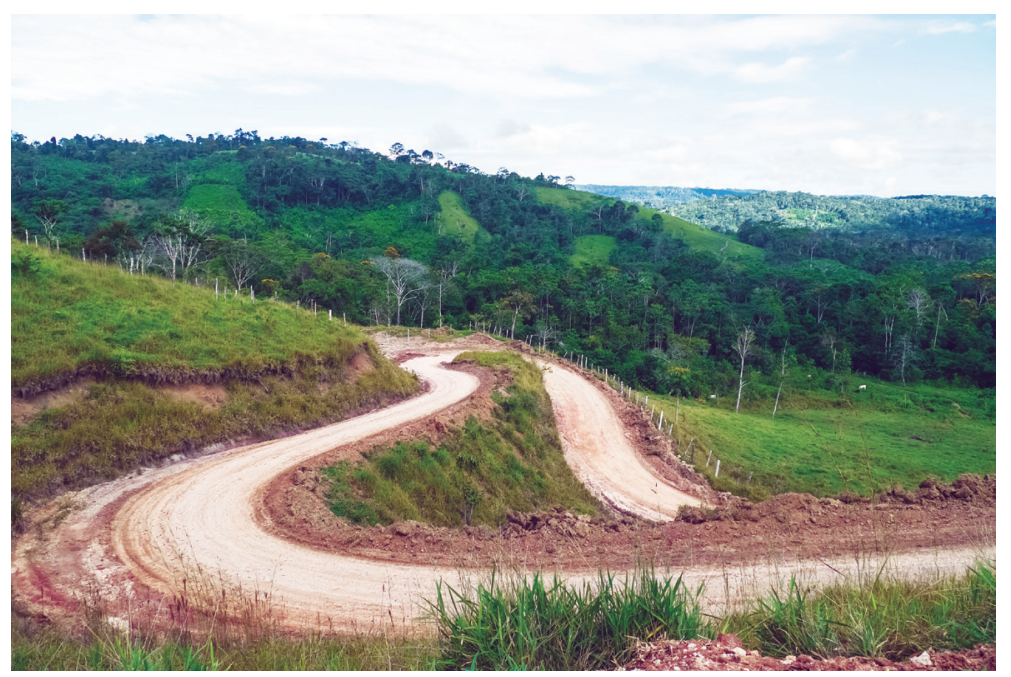

Figura 11. Tramo de la carretera desde Ponchoni hasta Oventeni Archivo Liliana Fernández, 2012.

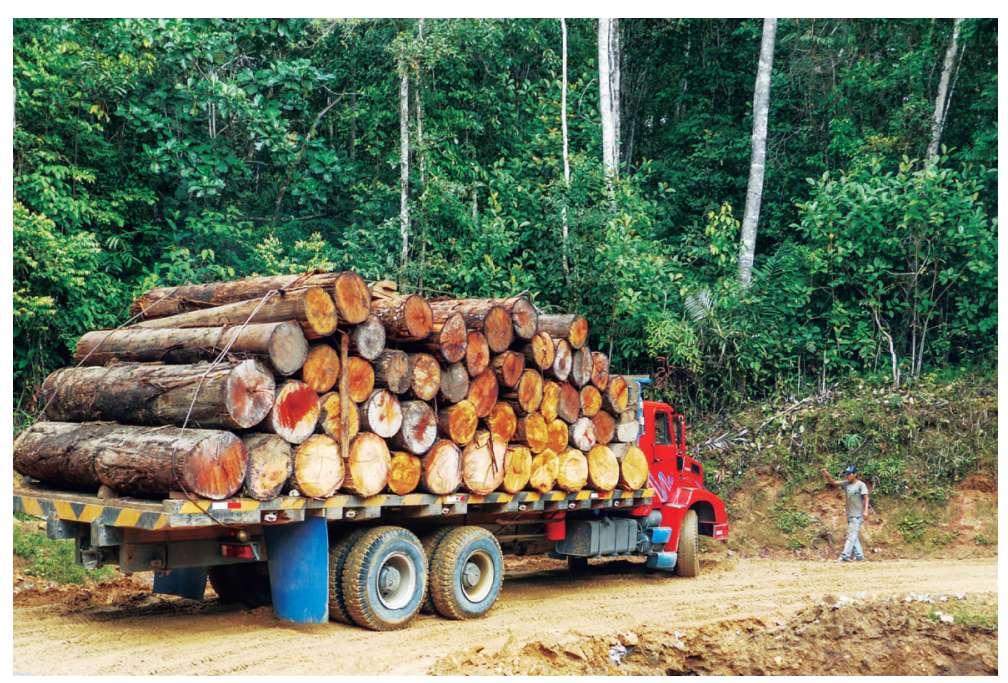

Figura 12. Transporte de madera en el Gran Pajonal Archivo Liliana Fernández, 2012. 
El desarrollo económico ha alterado la organización del espacio y del tiempo en el Gran Pajonal. El territorio se está transformando: hay comunidades que ya no tienen recursos propios, las chacras han disminuido dando paso a extensos cafetales o pajonales para el ganado. Las necesidades económicas como estudiar, curar alguna enfermedad e incluso alimentarse han generado nuevos proyectos de vida y la obligación de pensar no solo en un futuro inmediato, sino en actividades sustentables a través del tiempo. Hay que entender, sin embargo, que el desarrollo económico en el Gran Pajonal es irregular. No todos los ashéninkas se han beneficiado de los últimos proyectos por modernizar Oventeni y las comunidades nativas. El acceso a la modernidad depende de las metas y motivaciones personales, de factores logísticos, de voluntad política, de recursos comunales, entre otros.

\section{Conclusión}

La conciencia del valor del Gran Pajonal se vio fortalecida, en un primer momento, por el proceso de titulación de comunidades nativas y, posteriormente, por el proceso de distritalización, aunque de manera distinta. La titulación de comunidades nativas implicaba recuperar el keshii, fortalecer la identidad. En cambio, el proceso de distritalización generó dudas, temor de perder territorio, reflexión sobre el desarrollo y apertura al mercado nacional. Esto ha generado que la construcción de la identidad étnica se consolide sobre la base del sentido de pertenencia al keshii, al territorio pajonalino. Los distintos sentimientos que puede generar la posesión o pérdida del territorio desencadenan el proceso de construcción de la identidad frente a los mestizos e inclusive los asháninkas.

Los procesos de desarrollo fomentados por los líderes ashéninkas y autoridades colonas han generado modificaciones en la geografía del keshii, en los estilos de vida ashéninka, en la organización social dentro y fuera de la comunidad. Aunque el impacto es desigual y el desarrollo no es uniforme, desde el centro se viene generando un nuevo modelo de vida, un nuevo patrón cultural, cuyos efectos sobre la visión del territorio no se pueden pronosticar con exactitud, pero que sí permiten afirmar que la valoración del territorio descansa sobre ese sentido de pertenencia al espacio que constituye el seno de la cosmovisión ashéninka.

El aumento de mestizos debido a la creación de caseríos para crear el distrito acelerará el cambio sociocultural de las comunidades vecinas. No obstante, aunque el número de comunidades nativas ha aumentado y su extensión también, el dominio económico y la iniciativa de desarrollo parte de los mestizos y se evidencia en la infraestructura de Oventeni y los servicios que ofrece, de los cuales se benefician ellos en primer lugar. A pesar de esto, Oventeni es el centro político y económico para todos, incluido los ashéninkas, principalmente para aquellos que viven en las comunidades aledañas y que conforman el sector Oventeni. En cambio, no es un referente así de importante para las comunidades del sector carretera (la carretera Puerto Ocopa-Atalaya que cruza el Gran Pajonal). Debido a las distancias que las separan de Oventeni, estas comunidades tienen a las ciudades de Satipo y Atalaya como referentes inmediatos de un centro políticoeconómico.

La apropiación de Oventeni por parte de los ashéninkas se da en un sentido simbólico como el principal referente del Gran Pajonal fuera de sus límites, en la ciudad, frente al otro. Por ser modelo de desarrollo y ser lo más cercano a una ciudad, los jóvenes generalmente proyectan que algún día sus comunidades serán como Oventeni, mientras tanto manifiestan la pertenencia al centro cuando el discurso así lo exige. No obstante, esta referencia y sentido de pertenencia es contextual, depende de situaciones concretas. Internamente, la construcción de la identidad se 
basa en el sentido de pertenencia a las comunidades nativas que finalmente dan la categoría de comunero al ashéninka y legitiman la ciudadanía ashéninka y el derecho a poseer tierra.

La valoración del keshii, entonces, no depende de un lugar específico, sino del todo que va más allá de los límites distritales propuestos, se extiende más allá de lo real y concreto, ya que encuentra su sentido en la historia de un pueblo, se acuña en su memoria y desde ahí se fortalece y se consolida como la base de la construcción de la identidad ashéninka.

\section{Referencias}

Barth, F. (comp.). (1976). Los grupos étnicos y sus fronteras. La organización social de las diferencias culturales. México D. F.: Fondo de Cultura Económica.

Bello, A. (2004). Etnicidad y ciudadanía en América Latina. La acción colectiva de los pueblos indígenas. Santiago de Chile: CEPAL, GTZ.

Benavides, M. (2006). Atlas de comunidades nativas de la Selva Central. Lima: Instituto del Bien Común.

Cardoso, R. (2007). Etnicidad y estructura social. México: CIESAS, Universidad Autónoma Metropolitana, Universidad Iberoamericana.

Fernández, L. (2015). Territorio y memoria en el proceso de distritalización del Gran Pajonal (Ucayali-Perú). INDES 3(2), 29-36.

Fernández, L. (2017). Construcción identitaria de los jóvenes ashéninkas del Gran Pajonal en el siglo XXI. Tesis de maestría. UNMSM, Lima.

García, P. y Surralés A. (2009). Antropología de un derecho. Libre determinación territorial de los pueblos indígenas como derecho humano. Copenhague: IWGIA.

Hvalkof, S. y Veber H. (2005). Ashéninka. En Santos Granero, Fernando y Frederica Barclay (eds.) Guía etnográfica de la Alta Amazonia. Volumen V: Campa Ribereños/Ashéninka. Lima: Institut français d'études andines-IFEA; Smithsonian Tropical Research Institute.

Jenkins, R. (2008a). Rethinking ethnicity. Arguments and explorations. $2^{a}$ edición. Londres: Sage Publications.

Quintori, C. (2003). Proceso histórico de conformación de la Organización Ashéninka del Gran Pajonal. Tesis para optar el título de profesor en educación primaria intercultural bilingüe. Satipo: FORMABIAP.

Terrén, E. (2002). La etnicidad y sus formas: aproximación a un modelo completo de la pertenecía étnica. Revista Papers 66, 45-57. Barcelona: Universidad Autónoma de Barcelona.

Varese, S. (1973). La Sal de los Cerros. $2^{\circ}$ ed. Lima: Retablo de Papel.

Vilcapoma, J.C. (2017). Compendio, síntesis y matriz de competencias de la base legal de la normatividad vigente sobre los procesos de titulación de tierras en comunidades nativas de la Amazonía. Lima: Fondo Editorial UNALM. 